\title{
Spatiotemporal Dynamics Induced by Nonlocal Competition in a Diffusion Predator-Prey System with Habitat Complexity
}

\section{Ruizhi Yang}

Northeast Forestry University

Chenxuan Nie

Northeast Forestry University

Dan Jin ( $\square$ jindan720@163.com )

Northeast Forestry University

\section{Research Article}

Keywords: Predator-prey, Nonlocal competition, Delay, Hopf bifurcation

Posted Date: December 28th, 2021

DOI: https://doi.org/10.21203/rs.3.rs-1141642/v1

License: (c) (i) This work is licensed under a Creative Commons Attribution 4.0 International License.

Read Full License 


\title{
Spatiotemporal dynamics induced by nonlocal
}

\section{competition in a diffusion predator-prey system with}

\author{
habitat complexity \\ Ruizhi Yang, Chenxuan Nie, Dan Jin* \\ Department of Mathematics, Northeast Forestry University \\ Harbin 150040, Heilongjiang, China
}

December 22, 2021

\begin{abstract}
In this paper, we study a delayed diffusive predator-prey model with nonlocal competition in prey and habitat complexity. The local stability of coexisting equilibrium are studied by analyzing the eigenvalue spectrum. Time delay inducing Hopf bifurcation is investigated by using time delay as bifurcation parameter. We give some conditions for determining the bifurcation direction and the stability of the bifurcating periodic solution by utilizing the normal form method and center manifold theorem. Our results suggest that only nonlocal competition and diffusion together can induce stably spatial inhomogeneous bifurcating periodic solutions.
\end{abstract}

Keywords: Predator-prey; Nonlocal competition; Delay; Hopf bifurcation.

${ }^{*}$ Corresponding author. E-mail address: yangrz@nefu.edu.cn (R. Yang), jindan720@163.com (D. Jin) 


\section{Introduction}

Predator-prey model is an important model in biomathematics, which is used to study the growth law of two populations with predator-prey relationship [1-3]. In the ecological environment, habitat complexity plays an important role, which can be used to control the size and growth trend of the population. Many studies have shown that habitat complexity has a stabilizing effect on the predator-prey model [4-6]. In [8], Z. Ma and S. Wang studied a predator-prey model with habitat complexity and time delay, that is

$$
\left\{\begin{array}{l}
\frac{d u}{d t}=r u\left(1-\frac{u}{K}\right)--\frac{c(1-\beta) u^{\alpha} v}{1+\operatorname{ch}(1-\beta) u^{\alpha}} \\
\frac{d v}{d t}=\frac{e c(1-\beta) u^{\alpha}(t-\tau) v(t-\tau)}{1+\operatorname{ch}(1-\beta) u^{\alpha}(t-\tau)}-d v .
\end{array}\right.
$$

All parameters are positive. The biological description of parameters is in Table 1 . The authors assume there is a gestation delay in predator. In particular, parameter $\beta$ is used to measure the strength of habitat complexity, such as oyster and coral reefs, mangroves, sea grass beds and salt marshes [7]. The habitat complexity can be used to control the population growth. If $\alpha=1(\alpha=2)$, the functional response is Holling type I (II). In [8], Z. Ma and S. Wang studied the positivity, boundedness, stability, and Hopf bifurcation of the model (1.1). They observed the stabilizing and destabilizing effects of habitat complexity and periodic oscillation caused by time delay under some parameters.

In the ecological environment, the space is often inhomogeneous, and the spatial diffusion often occurs in population. Therefore, the reaction-diffusion equation may be more realistic when we study the predator-prey model. In addition, many scholars have studied the predator-prey model with reaction diffusion, and suggest that diffusion can induce spatial pattern, inhomogeneous periodic solution $[9,10]$. However, spatially homogeneous periodic solutions often appear in numerical simulation, which means the inhomogeneous periodic solutions are usually unstable. Therefore, we want to study the effect of diffusion term on the model (1.1), and whether inhomogeneous periodic solutions will appear? 
Table 1: Biological description of parameters in model (1.1).

\begin{tabular}{clcl}
\hline Parameter & Definition & Parameter & Definition \\
\hline$t$ & Time & $r$ & Prey intrinsic growth rate \\
$u(t)$ & Prey density & $v(t)$ & Predator density \\
$K$ & Prey carrying capacity & $c$ & Attack coefficient \\
$\beta$ & Strength of habitat complexity & $h$ & Handling time \\
$e$ & Conversion efficiency & $\tau$ & Gestation delay \\
$d$ & Death rate of predators & $\alpha$ & Positive real number \\
\hline
\end{tabular}

In fact, the resources is limited in nature, and competition within the population always exist. This competition is usually nonlocal. In $[11,12]$, the authors suggest that the consumption of resources in spatial location is related not only to the local population density, but also to the number of nearby population density. They measure this effect by weighting and integrating, modifing the $\frac{u}{K}$ as $\frac{1}{K} \int_{\Omega} G(x, y) u(y, t) d y . G(x, y)$ is some kernel function. In [13], the authors studied a diffusive predator-prey model with nonlocal competition effect, including stability of positive constant steady state and Hopf bifurcation. In [14] Geng et al. studied Hopf, Turing, double-Hopf, and Turing-Hopf bifurcations of a diffusive predator-prey model with nonlocal competition. In [15], Liu et al. studied a diffusive predator-prey model with nonlocal competition and time delay. These works show that spatially inhomogeneous bifurcating periodic solutions are stable, which is different from the model without nonlocal competition. Therefore, we want to study the effect of nonlocal competition on the model (1.1), and whether some new dynamic phenomena will appear?

Motivated by these, we incorporate nonlocal competition and spatial diffusion on the model 
(1.1), as follow

$$
\left\{\begin{array}{l}
\frac{\partial u(x, t)}{\partial t}=d_{1} \Delta u+r u\left(1-\frac{1}{K} \int_{\Omega} G(x, y) u(y, t) d y\right)-\frac{c(1-\beta) u^{\alpha} v}{1+c h(1-\beta) u^{\alpha}} \\
\frac{\partial v(x, t)}{\partial t}=d_{2} \Delta v+\frac{e c(1-\beta) u^{\alpha}(t-\tau) v(t-\tau)}{1+\operatorname{ch}(1-\beta) u^{\alpha}(t-\tau)}-d v, \quad x \in \Omega, t>0 \\
\frac{\partial u(x, t)}{\partial \bar{\nu}}=\frac{\partial v(x, t)}{\partial \bar{\nu}}=0, \quad x \in \partial \Omega, t>0 \\
u(x, \theta)=u_{0}(x, \theta) \geq 0, v(x, \theta)=v_{0}(x, \theta) \geq 0, \quad x \in \bar{\Omega}, \theta \in[-\tau, 0] .
\end{array}\right.
$$

Where $d_{1}$ and $d_{2}$ are diffusion coefficients of prey and predator. For the convenience of calculation, we choose $\Omega=(0, l \pi)$, where $l>0 . \frac{1}{K} \int_{\Omega} G(x, y) u(y, t) d y$ represents the nonlocal competition effect. The kernel function is

$$
G(x, y)=\frac{1}{|\Omega|}=\frac{1}{l \pi}, \quad x, y \in \Omega
$$

This is based on the assumption that the competition strength among prey individuals in the habitat is the same, that is the competition between any two prey is the same [14]. The boundary condition is Newman boundary, which is be explained by the fact that the habitat of the population is closed and no prey or predator can entering or leaving the habitat.

The aim of this paper is to study the effect of diffusion term and nonlocal competition on the model (1.2). The rest of this paper is organized as follows. In Sec. 2, we study the stability of coexisting equilibrium and existence of Hopf bifurcation. In Sec. 3, we study the Property of Hopf bifurcation. In Section 3, we give some numerical simulations to illustrate the theoretical results. In Sec. 4, we give a short conclusion.

\section{Stability analysis}

In this section, we will study the stability and existence of Hopf bifurcation at coexisting equilibrium. Denote $\mathbb{N}$ as positive integer set, and $\mathbb{N}_{0}$ as nonnegative integer set. $(0,0)$ and $(K, 0)$ are boundary equilibria of system (1.2). Make the following hypothesis

$\left(\mathbf{H}_{\mathbf{0}}\right)$

$$
e>\frac{d}{(1-\beta) c K^{\alpha}}+h d
$$


If $\left(\mathbf{H}_{\mathbf{0}}\right)$ holds, then system (1.2) has a unique coexisting equilibrium $E_{*}\left(u_{*}, v_{*}\right)$, where

$$
u_{*}=\left[\frac{c}{d}(1-\beta)(e-d h)\right]^{-\frac{1}{\alpha}}, \quad v_{*}=\frac{e r u_{*}\left(K-u_{*}\right)}{d K} .
$$

Linearize system $(1.2)$ at $E_{*}\left(u_{*}, v_{*}\right)$

$$
\frac{\partial u}{\partial t}\left(\begin{array}{c}
u(x, t) \\
u(x, t)
\end{array}\right)=D\left(\begin{array}{c}
\Delta u(t) \\
\Delta v(t)
\end{array}\right)+L_{1}\left(\begin{array}{c}
u(x, t) \\
v(x, t)
\end{array}\right)+L_{2}\left(\begin{array}{c}
u(x, t-\tau) \\
v(x, t-\tau)
\end{array}\right)+L_{3}\left(\begin{array}{c}
\hat{u}(x, t) \\
\hat{v}(x, t)
\end{array}\right),
$$

where

$$
\begin{aligned}
& D=\left(\begin{array}{cc}
d_{1} & 0 \\
0 & d_{2}
\end{array}\right), \quad L_{1}=\left(\begin{array}{cc}
a_{1} & a_{2} \\
0 & -d
\end{array}\right), \quad L_{2}=\left(\begin{array}{cc}
0 & 0 \\
b_{1} & d
\end{array}\right), \quad L_{3}=\left(\begin{array}{cc}
\hat{a} & 0 \\
0 & 0
\end{array}\right), \\
& a_{1}=r\left(1-\frac{u_{*}}{K}\right)-\frac{\alpha(1-\beta) c v_{*} u_{*}^{\alpha-1}}{\left((1-\beta) c h u_{*}^{\alpha}+1\right)^{2}}, \quad a_{2}=-\frac{(1-\beta) c u_{*}^{\alpha}}{(1-\beta) c h u_{*}^{\alpha}+1}<0, \\
& b_{1}=\frac{\alpha(1-\beta) \operatorname{cev} v_{*} u_{*}^{\alpha-1}}{\left(1+(1-\beta) \operatorname{ch} u_{*}^{\alpha}\right)^{2}}>0, \quad \hat{a}=-\frac{r u_{*}}{K}<0,
\end{aligned}
$$

and $\hat{u}=\frac{1}{l \pi} \int_{0}^{l \pi} u(y, t) d y$. The characteristic equation are

$$
\lambda^{2}+A_{n} \lambda+B_{n}+\left(C_{n}-d \lambda\right) e^{-\lambda \tau}=0, \quad n \in \mathbb{N}_{0},
$$

where

$$
\begin{aligned}
& A_{0}=-\left(a_{1}+\hat{a}-d\right), \quad B_{0}=-\left(a_{1}+\hat{a}\right) d, \quad C_{0}=\hat{a} d+a_{1} d-a_{2} b_{1}, \\
& A_{n}=\left(d_{1}+d_{2}\right) \frac{n^{2}}{l^{2}}-a_{1}+d, \quad B_{n}=d_{1} d_{2} \frac{n^{4}}{l^{4}}+\left(d d_{1}-a_{1} d_{2}\right) \frac{n^{2}}{l^{2}}-a_{1} d, \\
& C_{n}=-d d_{1} \frac{n^{2}}{l^{2}}+a_{1} d-a_{2} b_{1}, \quad n \in \mathbb{N} .
\end{aligned}
$$

\subsection{The case of $\tau=0$}

We will first discuss the stability of the coexisting equilibrium when the delay $\tau=0$. Make the following hypothesis

$\left(\mathbf{H}_{\mathbf{1}}\right) \quad a_{1}+\hat{a}<0, A_{n}-d>0, B_{n}+C_{n}>0$, for $n \in \mathbb{N}$,

$\left(\mathbf{H}_{2}\right) \quad a_{1}+\hat{a}<0, A_{k}-d<0$ (or $B_{k}+C_{k}<0$ ), for some $k \in \mathbb{N}$. 
Theorem 2.1. For system (1.2), assume $\tau=0$ and $\left(\mathbf{H}_{\mathbf{0}}\right)$ holds. If $\left(\mathbf{H}_{\mathbf{1}}\right)$ hold, $E_{*}\left(u_{*}, v_{*}\right)$ is locally asymptotically stable. If $\left(\mathbf{H}_{\mathbf{2}}\right)$ hold, $E_{*}\left(u_{*}, v_{*}\right)$ is Turing instable.

Proof. When $\tau=0$, the characteristic equations (2.3) are

$$
\lambda^{2}-\left(a_{1}+\hat{a}\right) \lambda-a_{2} b_{1}=0, \quad n=0 .
$$

and

$$
\lambda^{2}+\left(A_{n}-d\right) \lambda+B_{n}+C_{n}=0, \quad n \in \mathbb{N},
$$

If $\left(\mathbf{H}_{\mathbf{1}}\right)$ holds, we can obtain that the characteristic root of (2.5) and (2.6) all have negative real parts. Then $E_{*}\left(u_{*}, v_{*}\right)$ is locally asymptotically stable. If $\left(\mathbf{H}_{\mathbf{2}}\right)$ holds, we can obtain that the characteristic roots of (2.5) all have negative real parts. And the characteristic equation (2.6) at least have one root with positive real part. Then $E_{*}\left(u_{*}, v_{*}\right)$ is Turing unstable.

\section{$2.2 \quad$ The case of $\tau>0$}

Now, We will discuss the stability of the coexisting equilibrium when the delay $\tau>0$.

Lemma 2.1. Assume $\left(\mathbf{H}_{\mathbf{0}}\right)$ and $\left(\mathbf{H}_{\mathbf{1}}\right)$ hold. Eq. (2.3) has a pair of purely imaginary roots $\pm i \omega_{n}$ at $\tau_{n}^{j}, j \in \mathbb{N}_{0}, n \in \mathbb{S}$, where

$$
\omega_{n}=\sqrt{\frac{1}{2}\left[-\left(A_{n}^{2}-2 B_{n}-d^{2}\right)+\sqrt{\left(A_{n}^{2}-2 B_{n}-d^{2}\right)^{2}-4\left(B_{n}^{2}-C_{n}\right)}\right]}
$$

and

$$
\begin{aligned}
\tau_{n}^{j} & = \begin{cases}\frac{1}{\omega_{n}} \arccos \left(V_{\text {cos }}^{(n)}\right)+2 j \pi, & V_{\text {sin }}^{(n)} \geq 0, \\
\frac{1}{\omega_{n}}\left[2 \pi-\arccos \left(V_{\text {cos }}^{(n)}\right)\right]+2 j \pi, & V_{\text {sin }}^{(n)}<0 .\end{cases} \\
V_{\text {cos }}^{(n)} & =\frac{\omega^{2}\left(d A_{n}+C_{n}\right)-B_{n} C_{n}}{C_{n}^{2}+d^{2} \omega^{2}}, \quad V_{\text {sin }}^{(n)}=\frac{\omega\left(A_{n} C_{n}+B_{n} d-d \omega^{2}\right)}{C_{n}^{2}+d^{2} \omega^{2}}, \\
\mathbb{S} & =\left\{n \mid B_{n}-C_{n}<0, n \in \mathbb{N}_{0}\right\} .
\end{aligned}
$$


Proof. Let $\mathrm{i} \omega(\omega>0)$ be a solution of Eq. (2.3), then

$$
-\omega^{2}+\mathrm{i} \omega A_{n}+B_{n}+\left(C_{n}-d \mathrm{i} \omega\right)(\cos \omega \tau-\mathrm{i} \sin \omega \tau)=0
$$

We can obtain $\cos \omega \tau=\frac{\omega^{2}\left(d A_{n}+C_{n}\right)-B_{n} C_{n}}{C_{n}^{2}+d^{2} \omega^{2}}, \sin \omega \tau=\frac{\omega\left(A_{n} C_{n}+B_{n} d-d \omega^{2}\right)}{C_{n}^{2}+d^{2} \omega^{2}}$. It leads to

$$
\omega^{4}+\omega^{2}\left(A_{n}^{2}-2 B_{n}-d^{2}\right)+B_{n}^{2}-C_{n}^{2}=0 .
$$

Let $z=\omega^{2}$,then $(2.9)$ becomes

$$
z^{2}+z\left(A_{n}^{2}-2 B_{n}-d^{2}\right)+B_{n}^{2}-C_{n}^{2}=0
$$

and the roots of $(2.10)$ are $z^{ \pm}=\frac{1}{2}\left[-\left(A_{n}^{2}-2 B_{n}-d^{2}\right) \pm \sqrt{\left(A_{n}^{2}-2 B_{n}-d^{2}\right)^{2}-4\left(B_{n}^{2}-C_{n}\right)}\right]$. When $n \in \mathbb{S}$, if $\left(\mathbf{H}_{\mathbf{0}}\right)$ and $\left(\mathbf{H}_{\mathbf{1}}\right)$ hold, then $B_{n}+C_{n}>0\left(n \in \mathbb{N}_{0}\right), A_{0}^{2}-2 B_{0}-d^{2}=\left(\hat{a}+a_{1}\right)^{2}>0$, and $A_{n}^{2}-2 B_{n}-d^{2}=\left(a_{1}-d_{1} \frac{n^{2}}{l^{2}}\right)^{2}+d_{2} \frac{n^{2}}{l^{2}}\left(2 d+d_{2} \frac{n^{2}}{l^{2}}\right)>0$ for $n \in \mathbb{N}$. By direct calculation, we have $B_{0}-C_{0}=a_{2} b_{1}-2\left(\hat{a}+a_{1}\right) d$, and $B_{n}-C_{n}=d_{1} d_{2} \frac{n^{4}}{l^{4}}+\left(2 d d_{1}-a_{1} d_{2}\right) \frac{n^{2}}{l^{2}}+a_{2} b_{1}-2 a_{1} d$. Then $\mathbb{S}$ is a finite set, since $\lim _{n \rightarrow \infty}\left(B_{n}-C_{n}\right) \rightarrow+\infty$. It is easy to obtain $z^{+}>0$ and $z^{-}<0$ for $n \in \mathbb{S}$. Then eq. (2.3) has a pair of purely imaginary roots $\pm \mathrm{i} \omega_{n}$ at $\tau_{n}^{j}, j \in \mathbb{N}_{0}, n \in \mathbb{S}$.

Next, we verify the transversal condition for the existence of Hopf bifurcation.

Lemma 2.2. Assume $\left(\mathbf{H}_{\mathbf{0}}\right)$ and $\left(\mathbf{H}_{\mathbf{1}}\right)$ hold. Then $\operatorname{Re}\left[\left.\frac{d \lambda}{d \tau}\right|_{\tau=\tau_{n}^{j}}\right]>0$ for $n \in \mathbb{S}, j \in \mathbb{N}_{0}$.

Proof. By (2.3), we have

$$
\left(\frac{d \lambda}{d \tau}\right)^{-1}=\frac{2 \lambda+A_{n}-d e^{-\lambda \tau}}{\left(C_{n}-d \lambda\right) \lambda e^{-\lambda \tau}}-\frac{\tau}{\lambda}
$$

Then

$$
\begin{aligned}
{\left[\operatorname{Re}\left(\frac{d \lambda}{d \tau}\right)^{-1}\right]_{\tau=\tau_{n}^{j}} } & =\operatorname{Re}\left[\frac{2 \lambda+A_{n}-d e^{-\lambda \tau}}{\left(C_{n}-d \lambda\right) \lambda e^{-\lambda \tau}}-\frac{\tau}{\lambda}\right]_{\tau=\tau_{n}^{j}} \\
& =\left[\frac{1}{C_{n}^{2}+d^{2} \omega^{2}}\left(2 \omega^{2}+A_{n}^{2}-2 B_{n}-d^{2}\right)\right]_{\tau=\tau_{n}^{j}} \\
& =\left[\frac{1}{C_{n}^{2}+d^{2} \omega^{2}} \sqrt{\left(A_{n}^{2}-2 B_{n}-d^{2}\right)^{2}-4\left(B_{n}^{2}-C_{n}^{2}\right)}\right]_{\tau=\tau_{n}^{j}}>0 .
\end{aligned}
$$


Denote $\tau_{*}=\min \left\{\tau_{n}^{0} \mid n \in \mathbb{S}\right\}$. According to the above analysis, we have the following theorem.

Theorem 2.2. Assume $\left(\mathbf{H}_{\mathbf{0}}\right)$ and $\left(\mathbf{H}_{\mathbf{1}}\right)$ hold, then the following statements are true for system (1.2).

- $E_{*}\left(u_{*}, v_{*}\right)$ is locally asymptotically stable for $\tau \in\left[0, \tau_{*}\right)$.

- $E_{*}\left(u_{*}, v_{*}\right)$ is unstable for $\tau>\tau_{*}$.

- $\tau=\tau^{j}$ or $\tau_{n}^{j}\left(n \in \mathbb{S}, j \in \mathbb{N}_{0}\right)$ are Hopf bifurcation values of system (1.2).

\section{Property of Hopf bifurcation}

We give detailed computation about property of Hopf bifurcation using the method in $[16,17]$. For fixed $j \in \mathbb{N}_{0}$ and $n \in \mathbb{S}$, we denote $\tilde{\tau}=\tau_{n}^{j}$. Let $\bar{u}(x, t)=u(x, \tau t)-u_{*}$ and $\bar{v}(x, t)=$ $v(x, \tau t)-v_{*}$. Drop the bar, system (1.2) can be written as

$$
\left\{\begin{array}{l}
\frac{\partial u}{\partial t}=\tau\left[d_{1} \Delta u+r\left(u+u_{*}\right)\left(1-\frac{1}{K l \pi} \int_{0}^{l \pi}\left(u(y, t)+u_{*}\right) d y\right)-\frac{c(1-\beta)\left(u+u_{*}\right)^{\alpha}\left(v+v_{*}\right)}{1+c h(1-\beta)\left(u+u_{*}\right)^{\alpha}}\right], \\
\frac{\partial v}{\partial t}=\tau\left[d_{2} \Delta v+\frac{e c(1-\beta)\left(u(t-1)+u_{*}\right)^{\alpha}\left(v(t-1)+v_{*}\right)}{1+\operatorname{ch}(1-\beta)\left(u(t-1)+u_{*}\right)^{\alpha}}-d\left(v+v_{*}\right)\right] .
\end{array}\right.
$$

We rewrite system (3.1) as following system

$$
\left\{\begin{aligned}
\frac{\partial u}{\partial t}= & \tau\left[d_{1} \Delta u+a_{1} u+a_{2} v+\hat{a} \hat{u}+\alpha_{1} u^{2}+\hat{\alpha}_{1} u \hat{u}+\alpha_{2} u v+\alpha_{3} u^{3}+\alpha_{4} u^{2} v\right]+\text { h.o.t. } \\
\frac{\partial v}{\partial t}= & \tau\left[d_{2} \Delta v+b_{1} u(t-1)+d v(t-1)-d v+\beta_{1} u^{2}(t-1)+\beta_{2} u(t-1) v(t-1)\right. \\
& \left.+\beta_{3} u^{3}(t-1)+\beta_{4} u^{2}(t-1) v(t-1)\right]+ \text { h.o.t. }
\end{aligned}\right.
$$


where

$$
\begin{aligned}
& \alpha_{1}=-\frac{\alpha(\beta-1) c v_{*} u_{*}^{\alpha-2}\left(\alpha+(\alpha+1)(\beta-1) \operatorname{ch} u_{*}^{\alpha}-1\right)}{2\left((\beta-1) \operatorname{ch} u_{*}^{\alpha}-1\right)^{3}}, \quad \hat{\alpha}_{1}=\frac{-r}{K}, \quad \alpha_{2}=\frac{\alpha(\beta-1) c u_{*}^{\alpha-1}}{\left((\beta-1) \operatorname{ch} u_{*}^{\alpha}-1\right)^{2}}, \\
& \alpha_{3}=\frac{\alpha(\beta-1) c v_{*} u_{*}^{\alpha-3}\left(\alpha^{2}-3 \alpha+\left(\alpha^{2}+3 \alpha+2\right)(\beta-1)^{2} c^{2} h^{2} u_{*}^{2 \alpha}+4\left(\alpha^{2}-1\right)(\beta-1) \operatorname{ch} u_{*}^{\alpha}+2\right)}{6\left((\beta-1) c h u_{*}^{\alpha}-1\right)^{4}}, \\
& \alpha_{4}=-\frac{\alpha(\beta-1) c u_{*}^{\alpha-2}\left(\alpha+(\alpha+1)(\beta-1) \operatorname{ch} u_{*}^{\alpha}-1\right)}{2\left((\beta-1) \operatorname{ch} u_{*}^{\alpha}-1\right)^{3}}, \\
& \beta_{1}=\frac{\alpha(\beta-1) \operatorname{cev} v_{*} u_{*}^{\alpha-2}\left(\alpha+(\alpha+1)(\beta-1) \operatorname{ch} u_{*}^{\alpha}-1\right)}{2\left((\beta-1) \operatorname{ch} u_{*}^{\alpha}-1\right)^{3}}, \quad \beta_{2}=-\frac{\alpha(\beta-1) c e u_{*}^{\alpha-1}}{\left((\beta-1) c h u_{*}^{\alpha}-1\right)^{2}}, \\
& \beta_{3}=-\frac{\alpha(\beta-1) \operatorname{cev} u_{*}^{\alpha-3}\left(\alpha^{2}-3 \alpha+\left(\alpha^{2}+3 \alpha+2\right)(\beta-1)^{2} c^{2} h^{2} u_{*}^{2 \alpha}+4\left(\alpha^{2}-1\right)(\beta-1) \operatorname{ch} u_{*}^{\alpha}+2\right)}{6\left((\beta-1) \operatorname{ch} u_{*}^{\alpha}-1\right)^{4}}, \\
& \beta_{4}=\frac{\alpha(\beta-1) \operatorname{ce} u_{*}^{\alpha-2}\left(\alpha+(\alpha+1)(\beta-1) \operatorname{ch} u_{*}^{\alpha}-1\right)}{2\left((\beta-1) \operatorname{ch} u_{*}^{\alpha}-1\right)^{3}}
\end{aligned}
$$

Define the real-valued Sobolev space $X:=\left\{(u, v)^{T}: u, v \in H^{2}(0, l \pi),\left.\left(u_{x}, v_{x}\right)\right|_{x=0, l \pi}=0\right\}$, the complexification of $X \quad X_{\mathbb{C}}:=X \oplus \mathrm{i} X=\left\{x_{1}+\mathrm{i} x_{2} \mid x_{1}, x_{2} \in X\right\}$. and the inner product $<\tilde{u}, \tilde{v}>:=\int_{0}^{l \pi} \overline{u_{1}} v_{1} d x+\int_{0}^{l \pi} \overline{u_{2}} v_{2} d x$ for $\tilde{u}=\left(u_{1}, u_{2}\right)^{T}, \tilde{v}=\left(v_{1}, v_{2}\right)^{T}, \tilde{u}, \tilde{v} \in X_{\mathbb{C}}$. The phase space $\mathscr{C}:=C([-1,0], X)$ is with the $\sup$ norm, then we can write $\phi_{t} \in \mathscr{C}, \phi_{t}(\theta)=\phi(t+\theta)$ or $-1 \leq \theta \leq 0$. Denote $\beta_{n}^{(1)}(x)=\left(\gamma_{n}(x), 0\right)^{T}, \beta_{n}^{(2)}(x)=\left(0, \gamma_{n}(x)\right)^{T}$, and $\beta_{n}=\left\{\beta_{n}^{(1)}(x), \beta_{n}^{(2)}(x)\right\}$, where $\left\{\beta_{n}^{(i)}(x)\right\}$ is an an orthonormal basis of $X$. We define the subspace of $\mathscr{C}$ as $\mathbb{B}_{n}:=\operatorname{span}\{<$ $\left.\phi(\cdot), \beta_{n}^{(j)}>\beta_{n}^{(j)} \mid \phi \in \mathscr{C}, j=1,2\right\}, n \in \mathbb{N}_{0}$. There exists a $2 \times 2$ matrix function $\eta^{n}(\sigma, \tilde{\tau})$ $-1 \leq \sigma \leq 0$, such that $-\tilde{\tau} D \frac{n^{2}}{l^{2}} \phi(0)+\tilde{\tau} L(\phi)=\int_{-1}^{0} d \eta^{n}(\sigma, \tau) \phi(\sigma)$ for $\phi \in \mathscr{C}$. The bilinear form on $\mathscr{C}^{*} \times \mathscr{C}$ is defined by

$$
(\psi, \phi)=\psi(0) \phi(0)-\int_{-1}^{0} \int_{\xi=0}^{\sigma} \psi(\xi-\sigma) d \eta^{n}(\sigma, \tilde{\tau}) \phi(\xi) d \xi
$$

for $\phi \in \mathscr{C}, \psi \in \mathscr{C}^{*}$. Define $\tau=\tilde{\tau}+\mu$, then the system undergoes a Hopf bifurcation at $(0,0)$ when $\mu=0$, with a pair of purely imaginary roots $\pm \mathrm{i} \omega_{n_{0}}$. Let $A$ denote the infinitesimal generators of semigroup, and $A^{*}$ be the formal adjoint of $A$ under the bilinear form (3.3). Define the following function

$$
\delta\left(n_{0}\right)=\left\{\begin{array}{cc}
1 & n_{0}=0 \\
0 & n_{0} \in \mathbb{N} .
\end{array}\right.
$$


Choose $\eta_{n_{0}}(0, \tilde{\tau})=\tilde{\tau}\left[\left(-n_{0}^{2} / l^{2}\right) D+L_{1}+L_{3} \delta\left(n_{n_{0}}\right)\right], \eta_{n_{0}}(-1, \tilde{\tau})=-\tilde{\tau} L_{2}, \eta_{n_{0}}(\sigma, \tilde{\tau})=0$ for $-1<\sigma<$ 0 . Let $p(\theta)=p(0) e^{\mathrm{i} \omega_{n_{0}} \tilde{\tau} \theta}(\theta \in[-1,0]), q(\vartheta)=q(0) e^{-\mathrm{i} \omega_{n_{0}} \tilde{\tau} \vartheta}(\vartheta \in[0,1])$ be the eigenfunctions of $A(\tilde{\tau})$ and $A^{*}$ corresponds to $i \omega_{n_{0}} \tilde{\tau}$ respectively. We can choose $p(0)=\left(1, p_{1}\right)^{T}, q(0)=M\left(1, q_{2}\right)$, where $p_{1}=\frac{1}{a_{2}}\left(\mathrm{i} \omega_{n_{0}}+d_{1} n_{0}^{2} / l^{2}-a_{1}-\hat{a} \delta\left(n_{0}\right)\right), q_{2}=a_{2} /\left(\mathrm{i} \omega_{n_{0}}-d e^{\mathrm{i} \tau \omega_{n_{0}}}+d+\frac{\mathrm{d} 2 n^{2}}{l^{2}}\right)$, and $M=$ $\left(1+p_{1} q_{2}+\tilde{\tau}\left(q_{2} b_{1}+d p_{1} q_{2}\right) e^{-\mathrm{i} \omega_{n_{0}} \tilde{\tau}}\right)^{-1}$. Then (3.1) can be rewritten in an abstract form

$$
\frac{d U(t)}{d t}=(\tilde{\tau}+\mu) D \Delta U(t)+(\tilde{\tau}+\mu)\left[L_{1}\left(U_{t}\right)+L_{2} U(t-1)+L_{3} \hat{U}(t)\right]+F\left(U_{t}, \hat{U}_{t}, \mu\right),
$$

where

$$
F(\phi, \mu)=(\tilde{\tau}+\mu)\left(\begin{array}{c}
\alpha_{1} \phi_{1}^{2}(0)+\hat{\alpha}_{1} \phi_{1}(0) \hat{\phi}_{1}(0)+\alpha_{2} \phi_{1}(0) \phi_{2}(0)+\alpha \phi_{1}^{3}(0)+\alpha^{4} \phi_{1}^{2}(0) \phi_{2}(0) \\
\beta_{1} \phi_{1}^{2}(-1)+\beta_{2} \phi_{1}(-1) \phi_{2}(-1)+\beta \phi_{1}^{3}(-1)+\beta_{4} \phi_{1}^{2}(-1) \phi_{2}(-1)
\end{array}\right)
$$

respectively, for $\phi=\left(\phi_{1}, \phi_{2}\right)^{T} \in \mathscr{C}$ and $\hat{\phi}_{1}=\frac{1}{l \pi} \int_{0}^{l \pi} \phi d x$. Then the space $\mathscr{C}$ can be decomposed as $\mathscr{C}=P \oplus Q$, where $P=\left\{z p \gamma_{n_{0}}(x)+\bar{z} \bar{p} \gamma_{n_{0}}(x) \mid z \in \mathbb{C}\right\}, Q=\left\{\phi \in \mathscr{C} \mid\left(q \gamma_{n_{0}}(x), \phi\right)=\right.$ 0 and $\left.\left(\bar{q} \gamma_{n_{0}}(x), \phi\right)=0\right\}$. Then, system (3.6) can be rewritten as $U_{t}=z(t) p(\cdot) \gamma_{n_{0}}(x)+$ $\bar{z}(t) \bar{p}(\cdot) \gamma_{n_{0}}(x)+\omega(t, \cdot)$ and $\hat{U}_{t}=\frac{1}{l \pi} \int_{0}^{l \pi} U_{t} d x$, where

$$
z(t)=\left(q \gamma_{n_{0}}(x), U_{t}\right), \omega(t, \theta)=U_{t}(\theta)-2 \operatorname{Re}\left\{z(t) p(\theta) \gamma_{n_{0}}(x)\right\}
$$

then, we have $\dot{z}(t)=\mathrm{i} \omega) n_{0} \tilde{\tau} z(t)+\bar{q}(0)<F\left(0, U_{t}\right), \beta_{n_{0}}>$. There exists a center manifold $\mathcal{C}_{0}$ and $\omega$ can be written as follow near $(0,0)$.

$$
\omega(t, \theta)=\omega(z(t), \bar{z}(t), \theta)=\omega_{20}(\theta) \frac{z^{2}}{2}+\omega_{11}(\theta) z \bar{z}+\omega_{02}(\theta) \frac{\bar{z}^{2}}{2}+\cdots
$$

Then, restrict the system to the center manifold is $\dot{z}(t)=\mathrm{i} \omega_{n_{0}} \tilde{\tau} z(t)+g(z, \bar{z})$. Denote $g(z, \bar{z})=$ $g_{20} \frac{z^{2}}{2}+g_{11} z \bar{z}+g_{02} \frac{\bar{z}^{2}}{2}+g_{21} \frac{z^{2} \bar{z}}{2}+\cdots$. By direct computation, we have

$$
\begin{gathered}
g_{20}=2 \tilde{\tau} M\left(\varsigma_{1}+q_{2} \varsigma_{2}\right) I_{3}, \quad g_{11}=\tilde{\tau} M\left(\varrho_{1}+q_{2} \varrho_{2}\right) I, \quad g_{02}=\bar{g}_{20}, \\
g_{21}=2 \tilde{\tau} M\left[\left(\kappa_{11}+q_{2} \kappa_{21}\right) I_{3}+\left(\kappa_{12}+q_{2} \kappa_{22}\right) I_{4}\right],
\end{gathered}
$$

where $I_{3}=\int_{0}^{l \pi} \gamma_{n_{0}}^{3}(x) d x, I_{4}=\int_{0}^{l \pi} \gamma_{n_{0}}^{4}(x) d x, \varsigma_{1}=\alpha_{1}+\hat{\alpha}_{1} \delta\left(n_{0}\right)+\alpha_{2} p_{1}, \varsigma_{2}=e^{-2 i \tau \omega_{n_{0}}}\left(\beta_{1}+\beta_{2} p_{1}\right)$, $\varrho_{1}=\frac{1}{2} \alpha_{1}+\frac{1}{2} \hat{\alpha}_{1} \delta\left(n_{0}\right)+\frac{1}{4} \alpha_{2}\left(\bar{p}_{1}+p_{1}\right), \varrho_{2}=\frac{1}{2} \beta_{1}+\frac{1}{4} \beta_{2}\left(p_{1}+\bar{p}_{1}\right), \kappa_{11}=2 \omega_{11}^{(1)}(0)\left(2 \alpha_{1}+\hat{\alpha}_{1} \delta\left(n_{0}\right)+\right.$ 
$\left.\hat{\alpha}_{1}+\alpha_{2} p_{1}\right)+\omega_{20}^{(1)}(0)\left(2 \alpha_{1}+\hat{\alpha}_{1} \delta\left(n_{0}\right)+\hat{\alpha}_{1}+\alpha_{2} \bar{p}_{1}\right)+2 \alpha_{2} \omega_{11}^{(2)}(0)+\alpha_{2} \omega_{20}^{(2)}(0), \kappa_{12}=\frac{3 \alpha_{3}}{2}+\frac{\alpha_{4} \bar{p}_{1}}{2}+$ $\left.\alpha_{4} p_{1}, \kappa_{21}=2 \omega_{11}^{(} 1\right)(-1)\left(2 \beta_{1}+\beta_{2} p_{1}\right) e^{-i \tilde{\tau} \omega_{n_{0}}}+2 \beta_{2} \omega_{11}^{(2)}(-1) e^{-i \tilde{\tau} \omega_{n_{0}}}+\omega_{20}^{(1)}(-1)\left(2 \beta_{1}+\beta_{2} \bar{p}_{1}\right) e^{i \tilde{\tau} \omega_{n_{0}}}+$ $\beta_{2} \omega_{20}^{(2)}(-1) e^{i \tilde{\tau} \omega_{n_{0}}}, \kappa_{22}=\frac{1}{2} e^{-i \tilde{\tau} \omega_{n_{0}}}\left(3 \beta_{3}+\beta_{4}\left(\bar{p}_{1}+2 p_{1}\right)\right)$.

Now, we compute $W_{20}(\theta)$ and $W_{11}(\theta)$ for $\theta \in[-1,0]$ to give $g_{21}$. By $(3.7)$, we have

$$
\dot{\omega}=\dot{U}_{t}-\dot{z} p \gamma_{n_{0}}(x)-\dot{\bar{z}} \bar{p} \gamma_{n_{0}}(x)=A \omega+H(z, \bar{z}, \theta)
$$

where

$$
H(z, \bar{z}, \theta)=H_{20}(\theta) \frac{z^{2}}{2}+H_{11}(\theta) z \bar{z}+H_{02}(\theta) \frac{\bar{z}^{2}}{2}+\cdots
$$

Compare the coeffcients of (3.8) with (3.9), we have

$$
\left(A-2 \mathrm{i} \omega_{n_{0}} \tilde{\tau} I\right) \omega_{20}=-H_{20}(\theta), \quad A \omega_{11}(\theta)=-H_{11}(\theta) .
$$

Then, we have

$$
\begin{aligned}
& \omega_{20}(\theta)=\frac{-g_{20}}{\mathrm{i} \omega_{n_{0}} \tilde{\tau}} p(0) e^{\mathrm{i} \omega_{n_{0}} \tilde{\tau} \theta}-\frac{\bar{g}_{02}}{3 \mathrm{i} \omega_{n_{0}} \tilde{\tau}} \bar{p}(0) e^{-\mathrm{i} \omega_{n_{0}} \tilde{\tau} \theta}+E_{1} e^{2 \mathrm{i} \omega_{n_{0}} \tilde{\tau} \theta}, \\
& \omega_{11}(\theta)=\frac{g_{11}}{\mathrm{i} \omega_{n_{0}} \tilde{\tau}} p(0) e^{\mathrm{i} \omega_{n_{0}} \tilde{\tau} \theta}-\frac{\bar{g}_{11}}{\mathrm{i} \omega_{n_{0}} \tilde{\tau}} \bar{p}(0) e^{-\mathrm{i} \omega_{n_{0}} \tilde{\tau} \theta}+E_{2},
\end{aligned}
$$

where $E_{1}=\sum_{n=0}^{\infty} E_{1}^{(n)}, E_{2}=\sum_{n=0}^{\infty} E_{2}^{(n)}$,

$$
\begin{aligned}
& E_{1}^{(n)}=\left(2 \mathrm{i} \omega_{n_{0}} \tilde{\tau} I-\int_{-1}^{0} e^{2 \mathrm{i} \omega_{n_{0}} \tilde{\tau} \theta} d \eta_{n_{0}}(\theta, \bar{\tau})\right)^{-1}<\tilde{F}_{20}, \beta_{n}>, \\
& E_{2}^{(n)}=-\left(\int_{-1}^{0} d \eta_{n_{0}}(\theta, \bar{\tau})\right)^{-1}<\tilde{F}_{11}, \beta_{n}>, n \in \mathbb{N}_{0}, \\
& <\tilde{F}_{20}, \beta_{n}>=\left\{\begin{array}{ll}
\frac{1}{l \pi} \hat{F}_{20}, & n_{0} \neq 0, n=0, \\
\frac{1}{2 l \pi} \hat{F}_{20}, & n_{0} \neq 0, n=2 n_{0}, \\
\frac{1}{l \pi} \hat{F}_{20}, & n_{0}=0, n=0, \\
0, & \text { other, }
\end{array} \quad<\tilde{F}_{11}, \beta_{n}>= \begin{cases}\frac{1}{l \pi} \hat{F}_{11}, & n_{0} \neq 0, n=0, \\
\frac{1}{2 l \pi} \hat{F}_{11}, & n_{0} \neq 0, n=2 n_{0}, \\
\frac{1}{l \pi} \hat{F}_{11}, & n_{0}=0, n=0, \\
0, & \text { other, },\end{cases} \right.
\end{aligned}
$$

and $\hat{F}_{20}=2\left(\varsigma_{1}, \varsigma_{2}\right)^{T}, \hat{F}_{11}=2\left(\varrho_{1}, \varrho_{2}\right)^{T}$.

Thus, we can obtain

$$
\begin{aligned}
& c_{1}(0)=\frac{\mathrm{i}}{2 \omega_{n} \tilde{\tau}}\left(g_{20} g_{11}-2\left|g_{11}\right|^{2}-\frac{\left|g_{02}\right|^{2}}{3}\right)+\frac{1}{2} g_{21}, \quad \mu_{2}=-\frac{\operatorname{Re}\left(c_{1}(0)\right)}{\operatorname{Re}\left(\lambda^{\prime}(\tilde{\tau})\right)}, \\
& T_{2}=-\frac{1}{\omega_{n_{0}} \tilde{\tau}}\left[\operatorname{Im}\left(c_{1}(0)\right)+\mu_{2} \operatorname{Im}\left(\lambda^{\prime}\left(\tau_{n}^{j}\right)\right)\right], \quad \beta_{2}=2 \operatorname{Re}\left(c_{1}(0)\right) .
\end{aligned}
$$


Theorem 3.1. For any critical value $\tau_{n}^{j}\left(n \in \mathbb{S}, j \in \mathbb{N}_{0}\right)$, we have the following results.

- When $\mu_{2}>0$ (resp.<0), the Hopf bifurcation is forward (resp. backward).

- When $\beta_{2}<0$ (resp. $>0$ ), the bifurcating periodic solutions on the center manifold are orbitally asymptotically stable (resp. unstable).

- When $T_{2}>0$ (resp. $T_{2}<0$ ), the period increases (resp. decreases).

\section{Numerical simulations}

To analyze the effect of diffusion term and nonlocal competition, we compare our results with the work [8] and the following model without nonlocal competition.

$$
\left\{\begin{array}{l}
\frac{\partial u(x, t)}{\partial t}=d_{1} \Delta u+r u\left(1-\frac{u}{K}\right)-\frac{c(1-\beta) u^{\alpha} v}{1+c h(1-\beta) u^{\alpha}} \\
\frac{\partial v(x, t)}{\partial t}=d_{2} \Delta v+\frac{e c(1-\beta) u^{\alpha}(t-\tau) v(t-\tau)}{1+\operatorname{ch}(1-\beta) u^{\alpha}(t-\tau)}-d v, \quad x \in \Omega, t>0 \\
\frac{\partial u(x, t)}{\partial \bar{\nu}}=\frac{\partial v(x, t)}{\partial \bar{\nu}}=0, \quad x \in \partial \Omega, t>0 \\
u(x, \theta)=u_{0}(x, \theta) \geq 0, v(x, \theta)=v_{0}(x, \theta) \geq 0, \quad x \in \bar{\Omega}, \theta \in[-\tau, 0] .
\end{array}\right.
$$

$4.1 \quad \alpha=1$

Choose the same parameters with [8].

$$
d=1.16, K=300, c=0.23, h=0.054, e=0.115, \alpha=1, r=2.65 .
$$

Fix $d_{1}=0.1, d_{2}=0.1, l=0.5$, the bifurcation diagram of system (1.2) for $\beta$ and $\tau$ is in Fig 1. It shows that the habitat complexity has stabilizing effect which is consistent with [8]. However, with the increase of the habitat complexity, spatially inhomogeneous periodic solutions will appear.

If we choose $\beta=0.18$, then $\left(u_{*}, v_{*}\right)=(117.4610,18.7768)$ is a unique coexisting equilibrium. By direct computation, we have $\tau_{*}=\tau_{1}^{0} \approx 0.0883<\tau_{0}^{0} \approx 0.2860$. By Theorem 2.2 , we know that $E_{*}\left(u_{*}, v_{*}\right)$ is locally asymptotically stable when $\tau \in\left[0, \tau_{*}\right)$ (Fig. 2 left), which is consistent 


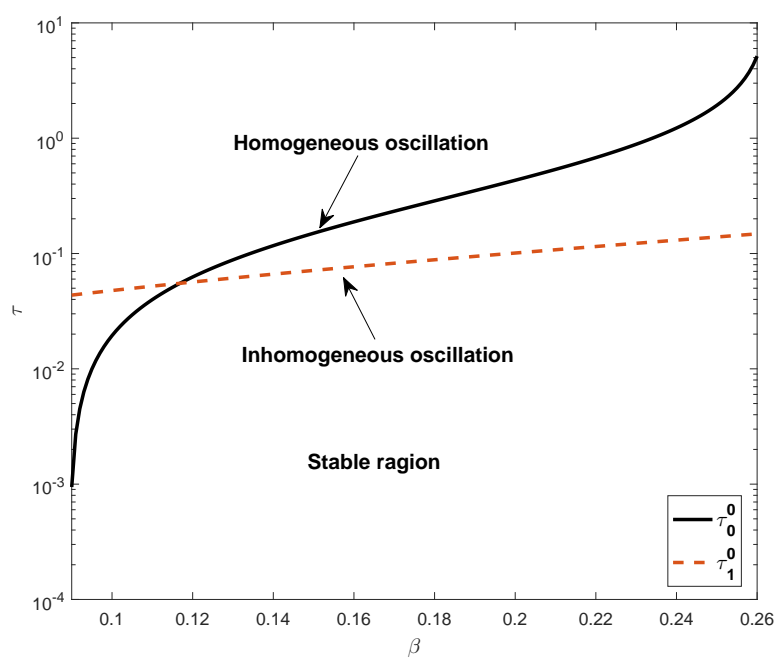

Figure 1: Bifurcation diagram of system (1.2) for $\beta$ and $\tau$.

with the results in [8] and for model (4.1) (Fig. 2 right). For model (1.2), Hopf bifurcation occurs when $\tau=\tau_{*}$. By Theorem 3.1, we have

$$
\mu_{2} \approx 1.6767 * 10^{-6}>0, \quad \beta_{2} \approx-2.0497 * 10^{-6}<0, \quad T_{2} \approx-4.3181 * 10^{-7}>0 .
$$

Hence, the stably spatially inhomogeneous bifurcating periodic solutions exist for $\tau>\tau_{*}$ (Fig. 3 left). This is inconsistent with the results in [8] and for model (4.1) (Fig. 3 right), where $\left(u_{*}, v_{*}\right)$ is local stable when $\tau=0.15$. When $\tau_{1}^{0}<\tau<\tau_{0}^{0}$, we can still observe stably spatially inhomogeneous bifurcating periodic solutions for model (1.2) (Fig. 4 left), but spatially homogeneous bifurcating periodic solutions for model (4.1) (Fig. 4 right). This show that only nonlocal competition and diffusion together can induce stably spatial inhomogeneous periodic solutions.

\section{$4.2 \alpha=2$}

Choose the same parameters with [8].

$$
d=1.16, K=300, c=0.045, h=0.054, e=0.115, \alpha=2, r=2.65 .
$$

Fix $d_{1}=0.3, d_{2}=0.1, l=1.2$, the bifurcation diagram of system (1.2) for $\beta$ and $\tau$ is in Fig 5. It shows that the habitat complexity has stabilizing effect which is consistent with [8]. 

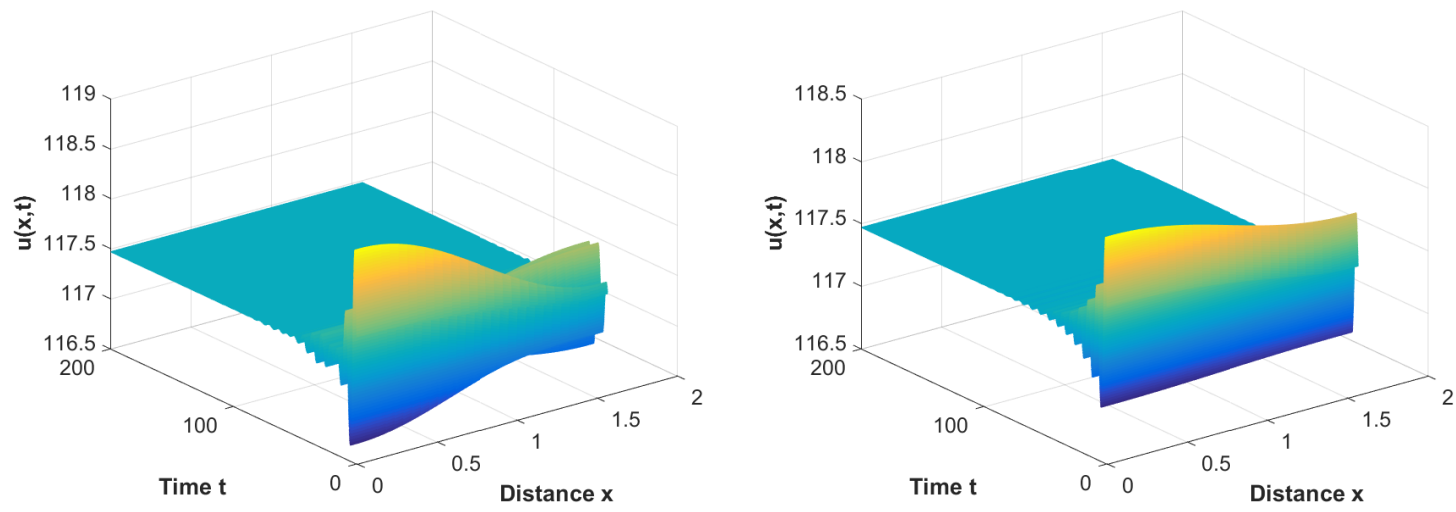

Figure 2: Numerical simulations for prey population with $\beta=0.18 \tau=0.04$. (Left: model (1.2). Right: model (4.1).
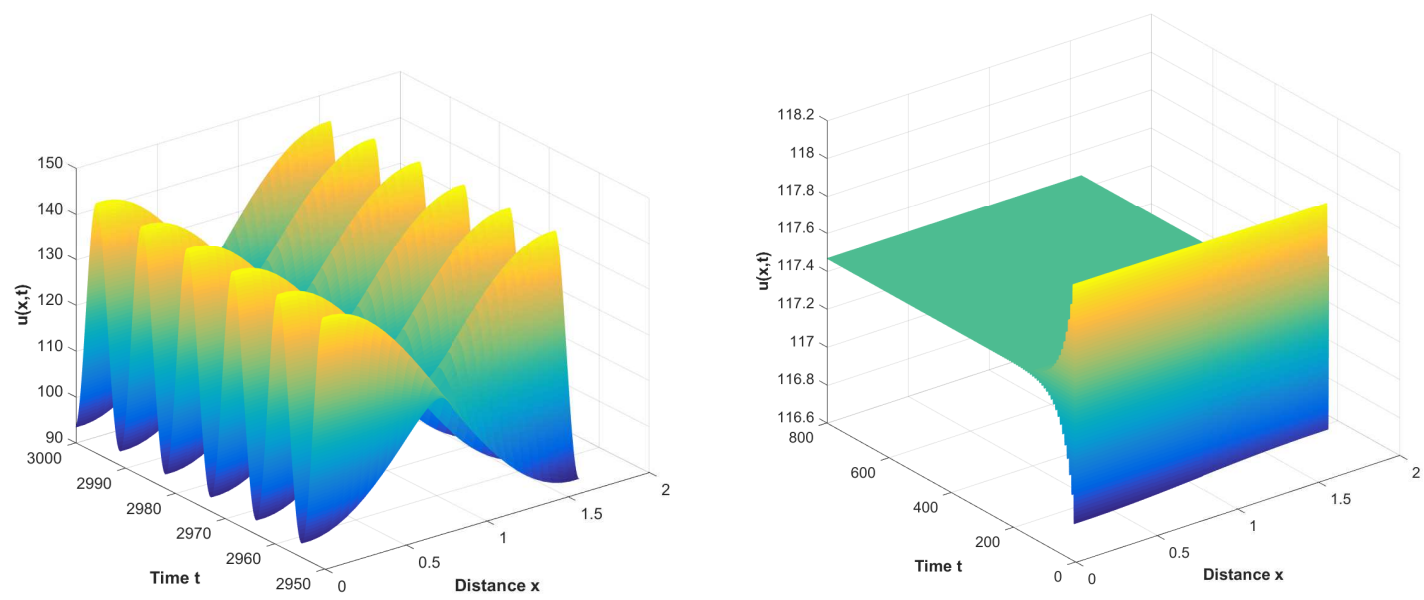

Figure 3: Numerical simulations for prey population with $\beta=0.18 \tau=0.15$. (Left: model (1.2).

Right: model (4.1). 

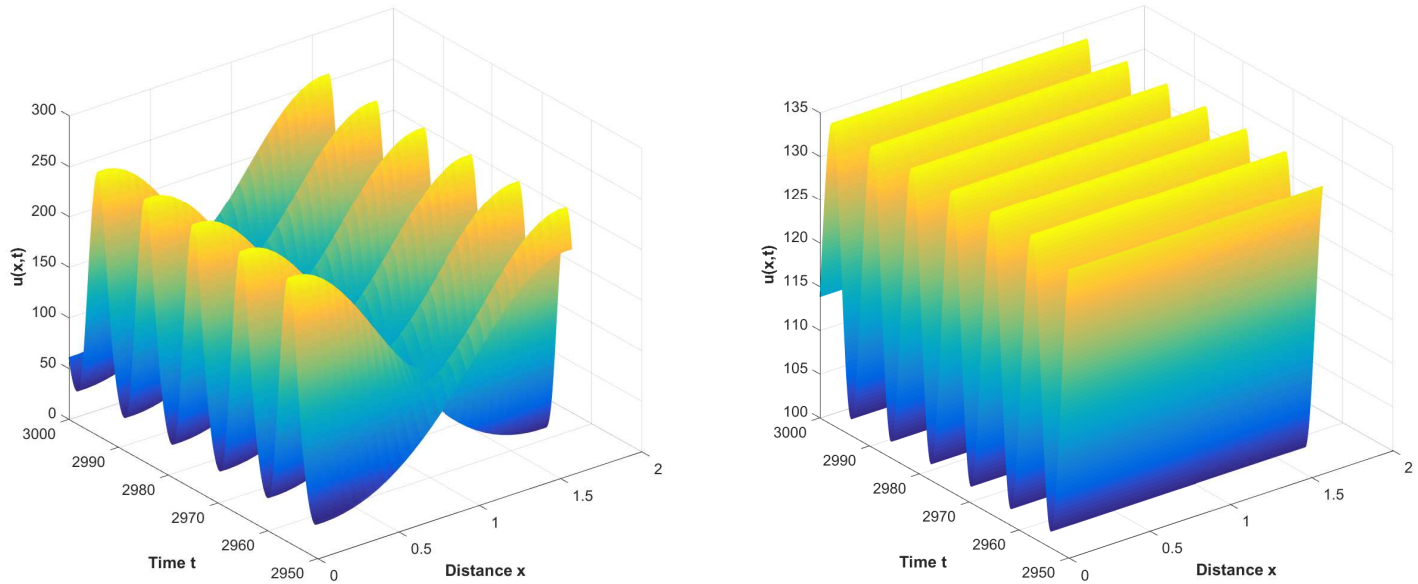

Figure 4: Numerical simulations for prey population with $\beta=0.18 \tau=0.3$. (Left: model (1.2). Right: model (4.1).

However, with the increase of the habitat complexity, spatially inhomogeneous periodic solutions will appear.

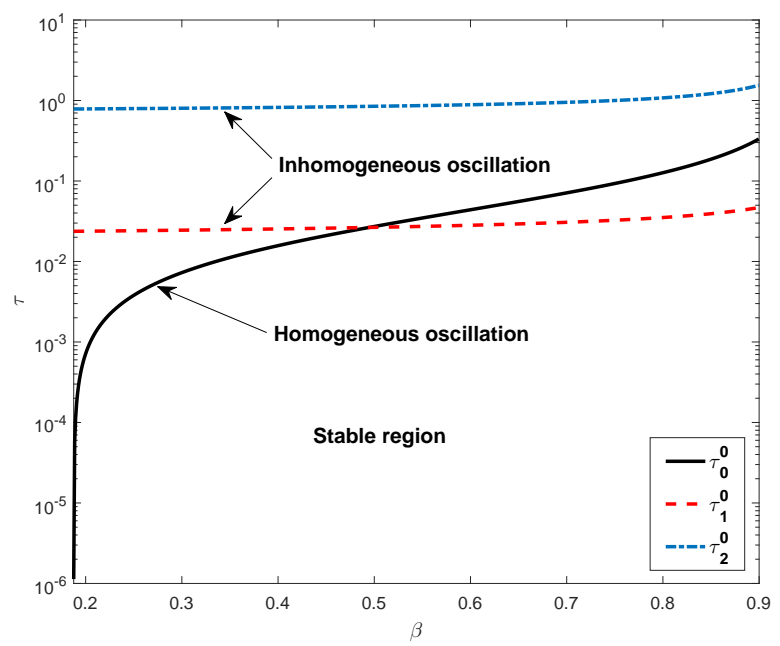

Figure 5: Bifurcation diagram of system (1.2) for $\beta$ and $\tau$.

We choose $\beta=0.68$, then $\left(u_{*}, v_{*}\right)=(39.2236,8.9574)$ is a unique coexisting equilibrium. Hypothesis $\left(\mathbf{H}_{\mathbf{0}}\right)$ and $\left(\mathbf{H}_{\mathbf{1}}\right)$ is satisfied. By direct computation, we have $\tau_{*}=\tau_{1}^{0} \approx 0.0301<$ $\tau_{0}^{0} \approx 0.0644$. By Theorem 2.2 , we know that $E_{*}\left(u_{*}, v_{*}\right)$ is locally asymptotically stable when 
$\tau \in\left[0, \tau_{*}\right)$ (Fig. 6 left), which is consistent with the results in [8] and for model (4.1) (Fig. 6 right). For model (1.2), Hopf bifurcation occurs when $\tau=\tau_{*}$. By Theorem 3.1, we have

$$
\mu_{2} \approx 2.1269 * 10^{-6}>0, \quad \beta_{2} \approx-4.81928 * 10^{-6}<0, \quad T_{2} \approx 5.6381 * 10^{-5}>0 .
$$

Hence, the stably spatially inhomogeneous bifurcating periodic solutions exist for $\tau>\tau_{*}$ (Fig. 7). This is inconsistent with the results in [8] and for model (4.1), in which $\left(u_{*}, v_{*}\right)$ is local stable with the same parameters (Fig. 7 right). When $\tau_{1}^{0}<\tau<\tau_{0}^{0}$, we can still observe stably spatially inhomogeneous bifurcating periodic solutions for model (1.2) (Fig. 8 left), but spatially homogeneous bifurcating periodic solutions for model (4.1) (Fig. 8 right).
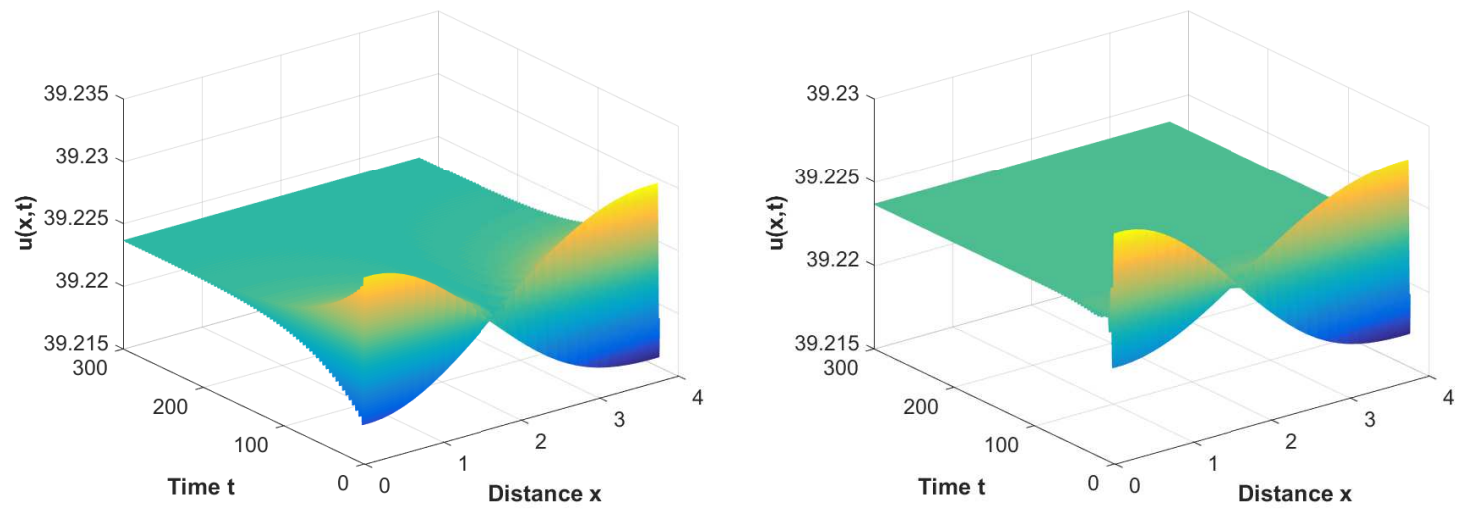

Figure 6: Numerical simulations for prey population with $\beta=0.68 \tau=0.02$. (Left: model (1.2). Right: model (4.1).

\section{$4.3 \alpha=3$}

Choose the same parameters with [8].

$$
d=1.16, K=30, c=0.24, h=0.054, e=0.07, \alpha=3, r=2.65 .
$$

Fix $d_{1}=0.5, d_{2}=0.1, l=0.75$, the bifurcation diagram of system (1.2) for $\beta$ and $\tau$ is in Fig 9. It shows that the habitat complexity has stabilizing effect which is consistent with [8].

Fix $\beta=0.68$, then $\left(u_{*}, v_{*}\right)=(12.7079,1.1714)$ is a unique coexisting equilibrium. By direct computation, we have $\tau_{*}=\tau_{1}^{0} \approx 0.0287<\tau_{0}^{0} \approx 0.1830$. By Theorem 2.2 , we know that 

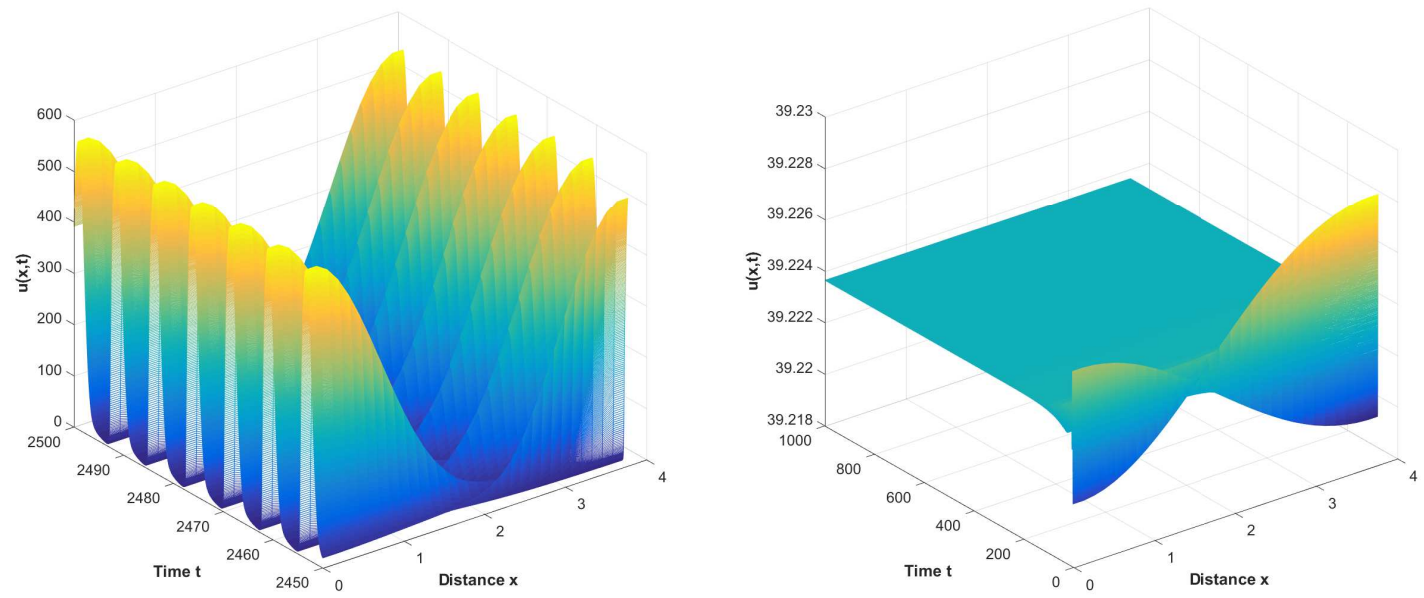

Figure 7: Numerical simulations for prey population with $\beta=0.68 \tau=0.04$. (Left: model (1.2). Right: model (4.1).
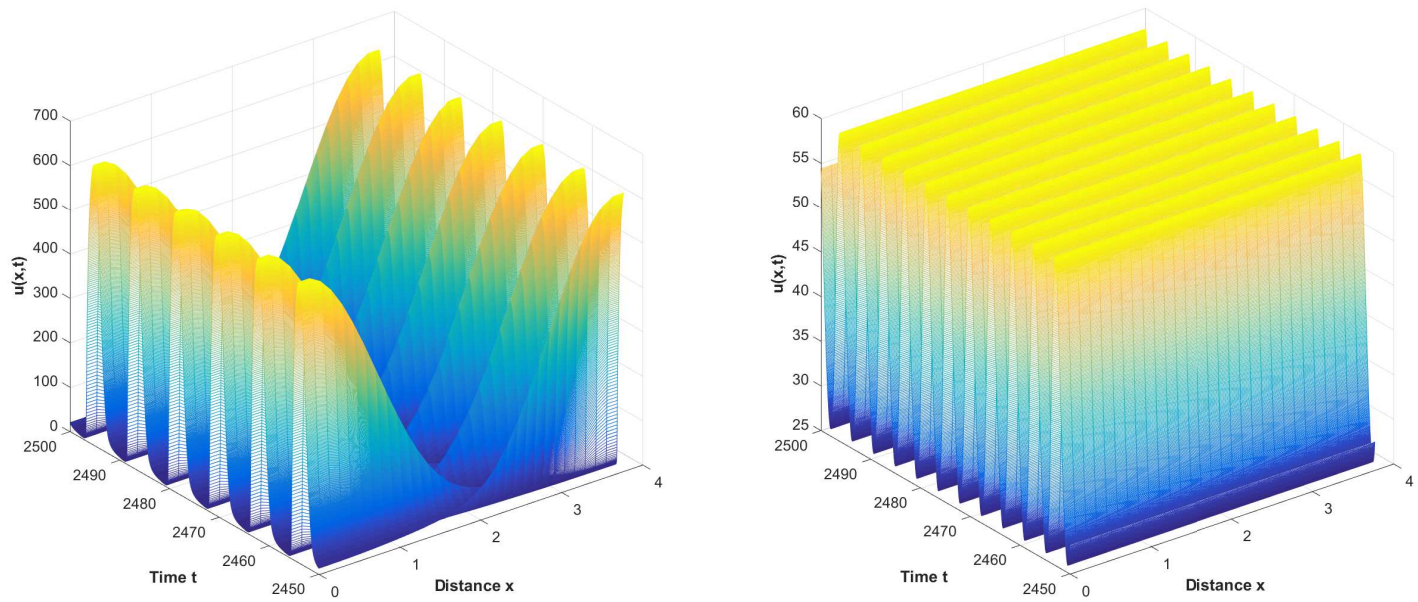

Figure 8: Numerical simulations for prey population with $\beta=0.68 \tau=0.07$. (Left: model (1.2).

Right: model (4.1). 


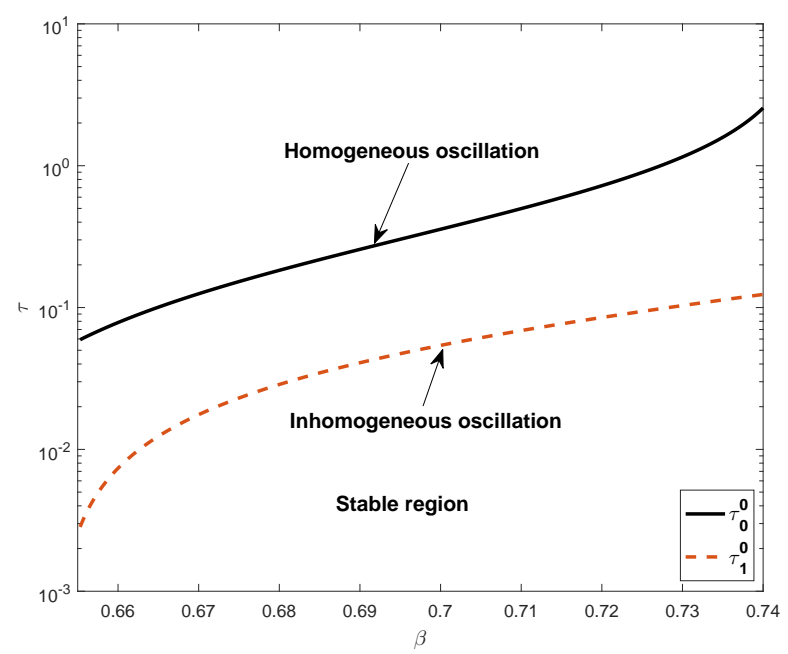

Figure 9: Bifurcation diagram of system (1.2) for $\beta$ and $\tau$.

$E_{*}\left(u_{*} \approx 12.7079, v_{*} \approx 1.1714\right)$ is locally asymptotically stable when $\tau \in\left[0, \tau_{*}\right)$ for models $(1.2)$ and (4.1) (Fig. 10). Hopf bifurcation occurs when $\tau=\tau_{*}$. By Theorem 3.1, we have

$$
\mu_{2} \approx 2.8642 * 10^{-4}>0, \quad \beta_{2} \approx-2.0057 * 10^{-4}<0, \quad T_{2} \approx-7.7547 * 10^{-4}<0 .
$$

Hence, the stably spatially inhomogeneous bifurcating periodic solutions exist for $\tau>\tau_{*}$ (Fig. 11 left). This is inconsistent with the results in [8] and for model (4.1), in which $E_{*}\left(u_{*}, v_{*}\right)$ is locally asymptotically stable (Fig. 11 right). When $\tau_{1}^{0}<\tau<\tau_{0}^{0}$, we can still observe stably spatially inhomogeneous bifurcating periodic solutions for model (1.2) (Fig. 12 left), but spatially homogeneous bifurcating periodic solutions for model (4.1) (Fig. 12 right).

\section{Conclusion}

In this paper, we study a delayed diffusive predator-prey model with nonlocal competition in prey and habitat complexity. We mainly study the local stability of coexisting equilibrium and existence of Hopf bifurcation. We also studied the property of bifurcating periodic solutions by the normal form method and center manifold theorem. Our work show that the habitat complexity has stabilizing effect when $\alpha=1,2,3$, which is consistent with [8]. Under the 

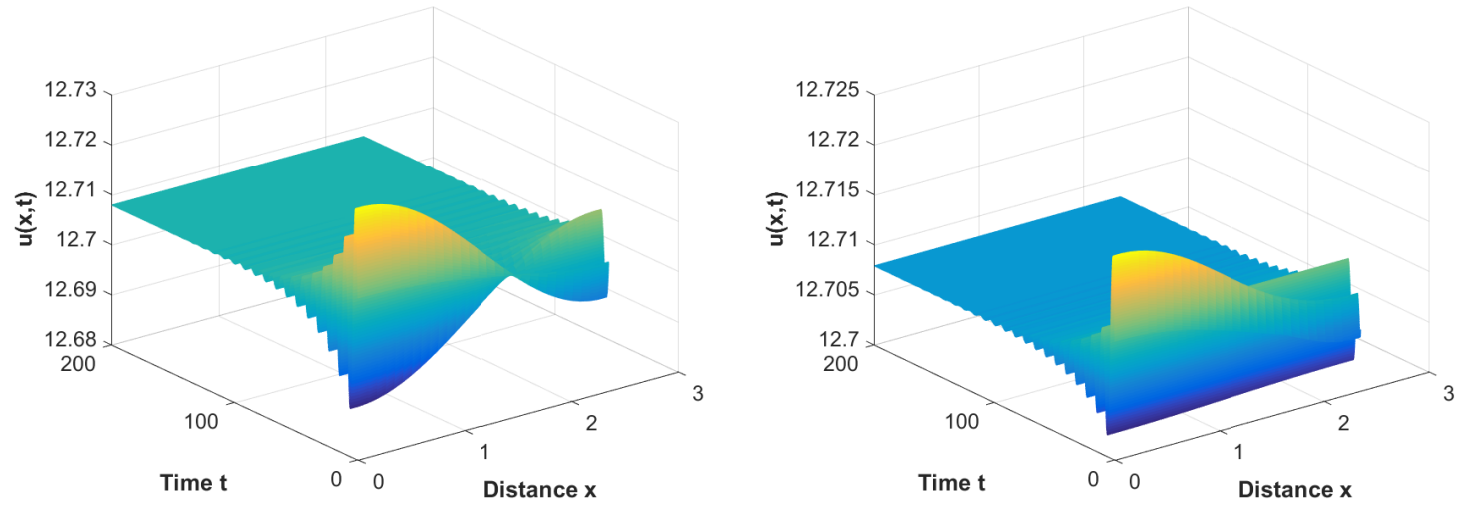

Figure 10: Numerical simulations for prey population with $\beta=0.68 \tau=0.01$. (Left: model (1.2). Right: model (4.1).
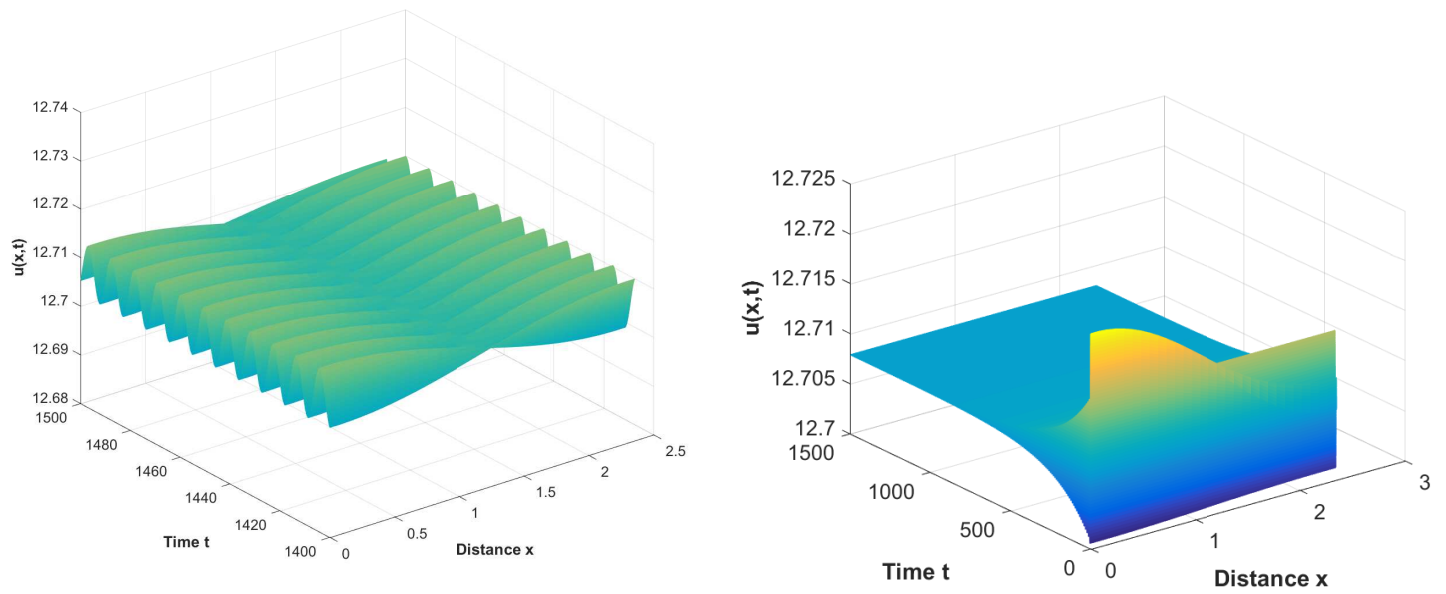

Figure 11: Numerical simulations for prey population with $\beta=0.68 \tau=0.15$. (Left: model (1.2). Right: model (4.1). 

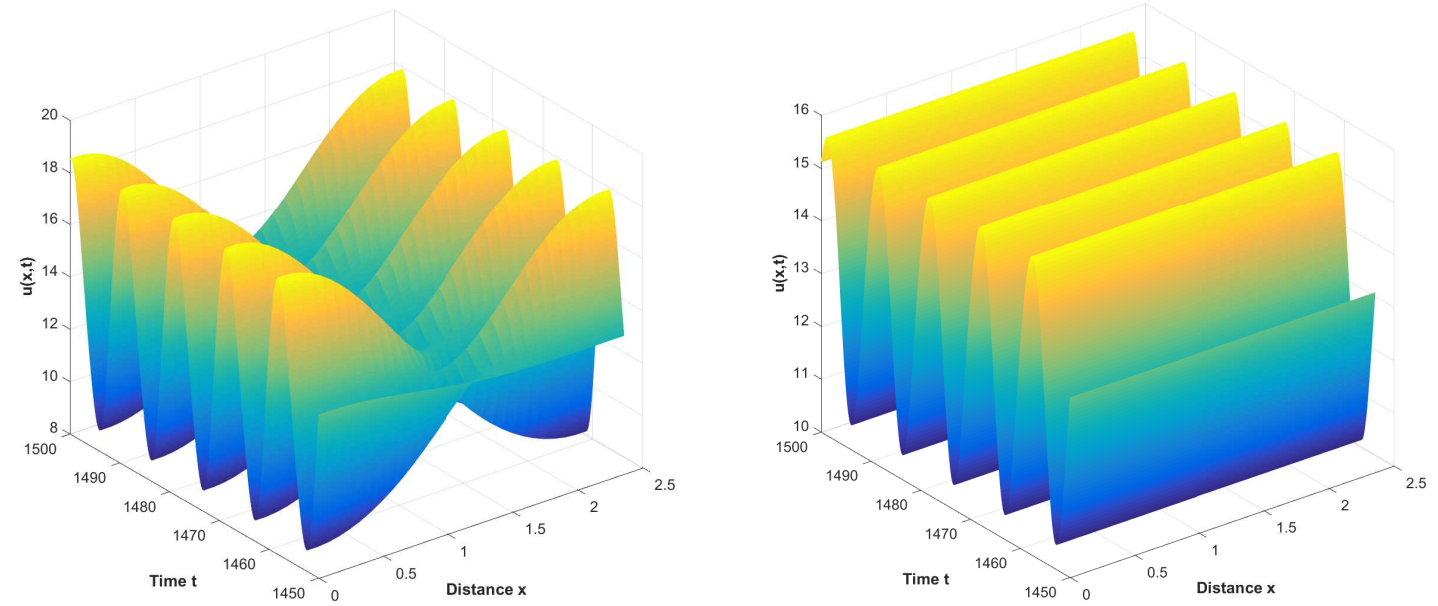

Figure 12: Numerical simulations for prey population with $\beta=0.68 \tau=0.35$. (Left: model (1.2). Right: model (4.1).

same parameters with [8], when $\beta$ cross some values, the stably spatial inhomogeneous periodic solutions will appear first when $\alpha=1,2$. Under some parameters, the inhomogeneous oscillation curve is always below the homogeneous oscillation curve when $\alpha=3$. Compared with the model (4.1), we conclude that only nonlocal competition and diffusion together can induce stably spatial inhomogeneous periodic solutions. In addition, the increase of time delay will increase the amplitude of the periodic solution.

\section{Statements and Declarations}

Funding This research is supported by the National Nature Science Foundation of China (No. 11601070) and Heilongjiang Provincial Natural Science Foundation (No. A2018001).

Competing Interests The authors have no relevant financial or non-financial interests to disclose. 
Author Contributions All authors contributed to the study conception and design. Material preparation, data collection and analysis were performed by Ruizhi Yang. Numerical simulations were performed by Ruizhi Yang and Chenxuan Nie. All authors read and approved the final manuscript.

Data Availability Data sharing is not applicable to this article as no datasets were generated or analyzed during the current study.

\section{References}

[1] Zhou Y, Yan X, Zhang C. Turing patterns induced by self-diffusion in a predator-prey model with schooling behavior in predator and prey. Nonlinear Dynamics, 2021, 105: $3731-3747$.

[2] Zhang, X, An, Q, Wang, L. Spatiotemporal dynamics of a delayed diffusive ratio-dependent predator-prey model with fear effect. Nonlinear Dynamics, 2021, 105: 3775-3790.

[3] Wang L , Zhang M, Jia M . A DELAYED PREDATOR-PREY MODEL WITH PREY POPULATION GUIDED ANTI-PREDATOR BEHAVIOUR AND STAGE STRUCTURE. Journal of Applied Analysis \& Computation, 2020, 11(4).

[4] Eklv, P. Effects of habitat complexity and prey abundance on the spatial and temporal distributions of perch (Perca fluviatilis) and pike (Esox lucius). Canadian Journal of Fisheries \& Aquatic Sciences, 1997, 54(54):1520-1531.

[5] August P V. The Role of Habitat Complexity and Heterogeneity. Ecology, 2008, 64(6):1495-1507.

[6] Canion C R, Heck K L. Effect of habitat complexity on predation success: re-evaluating the current paradigm in seagrass beds. Marine Ecology Progress, 2009, 393(393):37-46. 
[7] Jana D, Bairagi N. Habitat complexity, dispersal and metapopulations: Macroscopic study of a predator-prey system. Ecological Complexity, 2014, 17: 131-139.

[8] Ma Z, Wang S. A delay-induced predator-prey model with Holling type functional response and habitat complexity. Nonlinear Dynamics, 2018, 93: 1519-1544.

[9] Song Y., Peng Y., Zhang T. The spatially inhomogeneous Hopf bifurcation induced by memory delay in a memory-based diffusion system. Journal of DifferentialEquations, 2021, 300: 597-624.

[10] Yi F. Turing instability of the periodic solutions for reaction-diffusion systems with crossdiffusion and the patch model with cross-diffusion-like coupling. Journal of Differential Equations, 2021, 281:379-410.

[11] Britton N F. Aggregation and the competitive exclusion principle. Journal of Theoretical Biology, 1989, 136(1):57-66.

[12] Furter J, Grinfeld M. Local vs. non-local interactions in population dynamics. Journal of Mathematical Biology, 1989, 27(1):65-80.

[13] Chen S, Yu J. Stability and bifurcation on predator-prey systems with nonlocal prey competition, Discrete \& Continuous Dynamical Systems, 2018, 38 (1) : 43-62.

[14] Geng D, Jiang W, Lou Y, et al. Spatiotemporal patterns in a diffusive predator-prey system with nonlocal intraspecific prey competition. Studies in Applied Mathematics, 2021, 1-37.

[15] Liu Y, Duan D, Niu B. Spatiotemporal dynamics in a diffusive predator-prey model with group defense and nonlocal competition. Applied Mathematics Letters, 2020, 103: 106175.

[16] Wu J. Theory and Applications of Partial Functional Differential Equations. Springer Berlin, 1996. 
[17] Hassard B D, Kazarinoff N D, Wan Y H. Theory and applications of Hopf bifurcation. Cambridge University Press, Cambridge-New York, 1981. 